\title{
SIMPLIFIED COLLISION DETECTION IN 4R SERIAL MANIPULATORS
}

\author{
Artur HANDKE* \\ "Division of Machine Theory and Mechatronic Systems, Faculty of Mechanical Engineering, Wroclaw University of Technology, \\ ul. Łukasiewicza7/9 50-150 Wrocław, Poland \\ artur.handke@pwr.wroc.pl
}

\begin{abstract}
This paper concerns the problem of designating criteria for assessing the possibility of a collision between the elements of serial manipulators with three rotary joints allowing to flex the segments in common plane oriented by rotating the fourth connection. The issue of contactless and efficient functioning of described group of manipulators has been considered in the spatial system. Equations were derived determining the possibility of a collision between selected segments of both manipulators. Collision detection model isn't based on the information from the tactile sensors, but only on the relative position between the segments of manipulators. Based on the parameters defining the collision, the search for method of impossible collision was set on designing level, in order to minimize the time needed to examine all possible scenarios of collisions between segments. The results were included in development of methods and algorithms for planning and controlling movements of finger modules in anthropomorphic manipulator during grasping objects of indeterminate shape.
\end{abstract}

Key words: Serial Manipulator, Collision Detection, Movement Planning

\section{INTRODUCTION}

The problem of collision detection is an important aspect of the efficient execution of object manipulation process by group of serial manipulators. Collision is a particularly common situation in many operating spatial manipulators. An examined group of manipulators concerns fingers modules of an anthropomorphic hand type manipulator Hand-K3 (Handke and Twaróg, 2010) with adjustable orientation of the planes, in which three revolute joints operate. Since the collision detection cannot be based on tactile sensory, thus the simplest method to detect collision between moving segments' of discussed manipulators is to constantly determine the distance between them, based on their position in reference to the common system of coordinates. However, to minimize the time necessary to verify the established system of the collision criteria, the rationality of such solutions should be taken into account, due to energy consumption.

\section{TYPES OF COLLISIONS BETWEEN FINGER MODULES OF ANTHROPOMORPHIC MANIPULATOR IN SPATIAL SYSTEM}

In order to determine the parameters that clearly define the occurrence of a collision in the group of cooperating manipulators, two main criteria for collision detection were established. Discussed in this anthropomorphic manipulator Hand-K3 four combinations of subgroups consisting of three finger modules were set. It is related to the established algorithm for planning the movements of finger modules (Handke and Twaróg, 2012), in order to fully grasp the objects of indefinite shape, without the use of vision systems, as well as information from the tactile sensor network (De Luca, 2006). The collision in spatial system occurs in three directions, and due to the distinctive construction of the manipula- tor, it mainly occurs in a two-dimensional plane in which the movable segments revolute. This is only a simplification in order to separate the three-dimensional space from a plane containing the two directions of a collision in combination with the third direction, normal to this plane.

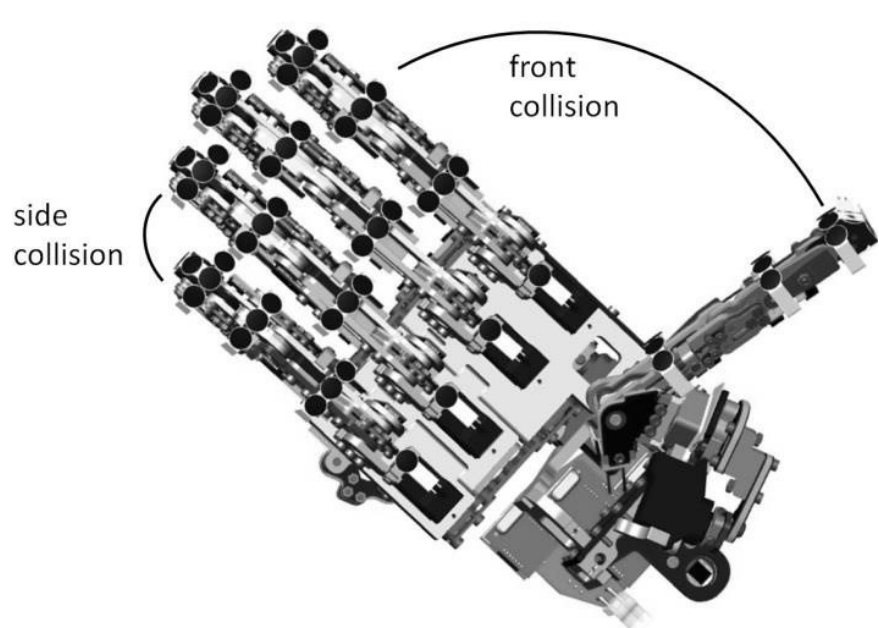

Fig. 1. Types of possible collisions in Hand-K3 anthropomorphic manipulator

The five-fingered manipulator of this type can have distinguished four pairs of fingers working in opposition to the thumb module to form four main planes in which front collision occurs (Fig. 1). Due to the possibility of variable orientation of the main planes of rotational finger modules in a limited range, it is necessary to define also a collision occurring between adjacent finger modules. Therefore, six pairs were separated among finger modules working initially in approximately parallel planes, to determine side collision. 


\section{PARAMETERIZATION OF FRONT AND SIDE COLLISIONS}

Analysis of the possibility of a collision was conducted by calculating the distance between the geometrical center of the selected segment of finger module (Fig. 2), and the plane that defines the type of collision. Analytical approach to this problem is often implemented by many authors (Bosscher, 2011). Because of the structural complexity of each segment, dimensions of the most distant surfaces of the m.i system of $m$-segment in $i$-finger module were assumed.

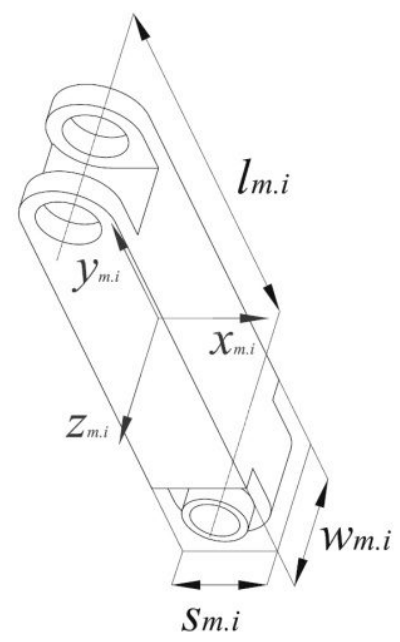

Fig. 2. Finger unit's segment basic dimensions

According to the proposed move control algorithm (Handke and Twaróg, 2012), collisions in finger modules are considered in a group of three among all five. Fig. 3 shows a selected group containing one finger $j$, set in opposition to the others.

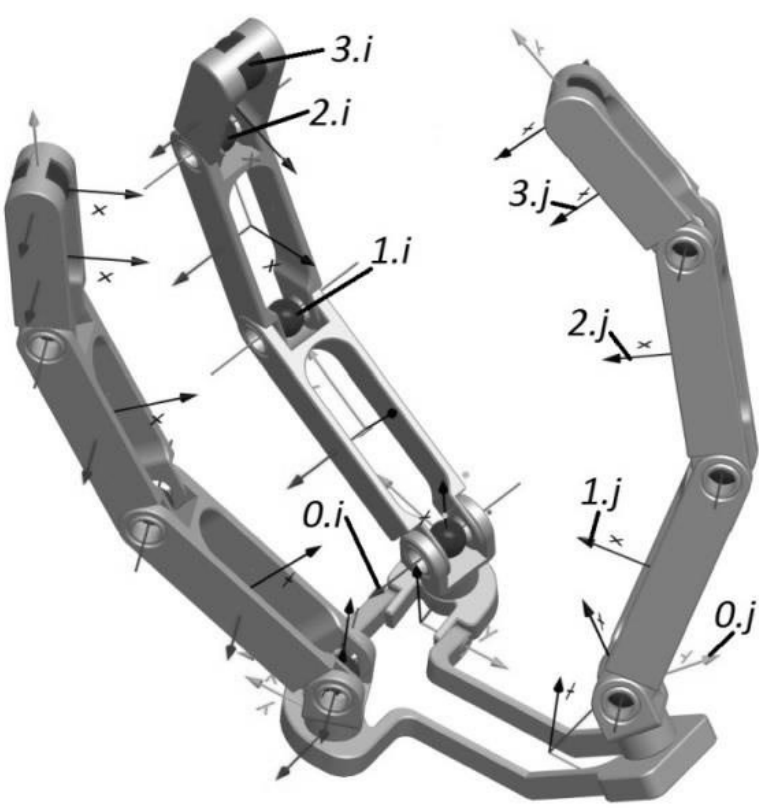

Fig. 3. Arrangement of the coordinates systems of the selected group of 3 finger modules

In order to create the parameters necessary to unambiguously identify a specific distance between the point and the plane, a general transformation matrix (1) between systems of $i$ and $j$ finger, was created, similar to the existing work (Spencer, 2008).I

$$
{ }^{m . i} A_{n . j}(t)={ }^{0 . j} A_{n . j}(t){ }^{0 . i} A_{0 . j}{ }^{m . i} A_{0 . i}(t)
$$

The transformation matrix between rotating joints that orients the main planes, in which all further segments flex, is conditionally fixed, according to the settings of rotations of $0 . j$ and $0 . i$. The rederivation of this matrix is necessary when the configuration of the main planes of joints $1 . i(j) \div 3 . i(j)$ is changed.

$$
f_{k}(A)=B \times A \times\left[\begin{array}{llll}
0 & 0 & 0 & 1
\end{array}\right]^{T}
$$

where: $\mathrm{k}$ - a specified coordinate of the Cartesian system

$$
k\left\{\begin{array}{l}
x \Rightarrow B=\left[\begin{array}{llll}
1 & 0 & 0 & 0
\end{array}\right] \\
y \Rightarrow B=\left[\begin{array}{llll}
0 & 1 & 0 & 0
\end{array}\right] \\
z \Rightarrow B=\left[\begin{array}{llll}
0 & 0 & 1 & 0
\end{array}\right]
\end{array}\right.
$$

To determine the $f_{k}$ distance searched in direction of the given system, relative to which it is established, the general form of the distance between the two systems of selected segments of finger modules was formed (2). Because $f_{k}$ distance is a scalar variable, depending on the selected direction, vector $B$ takes adequate form (3).

\subsection{Front collision}

The situation in which a collision between segments in the frontal plane of given segment is possible, may occur only for four pairs of opposing fingers. However, the situation in which two finger modules move in parallel planes occurs rarely in anthropomorphic manipulators with variable orientation of main planes. At this point, the situation with no front collision was omitted, due to the dimensional settings of the fingers.

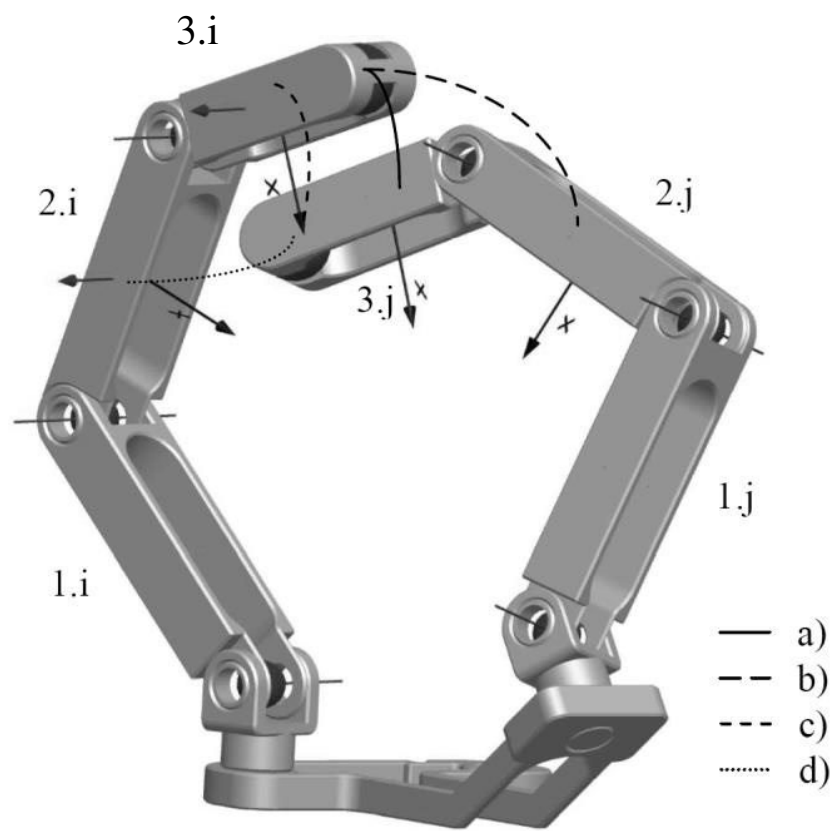

Fig. 4. Multiple front collision between opposite finger module's: a) front collision of $3 . i$ segment relative to $3 . j$ segment - 2 nd condition, b) front collision of 3.i segment relative to 2.j segment 1 st condition,c) front collision of $3 . j$ segment relative to $3 . i$ segment - 1st condition,d) front collision of 3.j segment relative to $2 . i$ segment - 1 st condition 
Two components of the $f_{k}$ parameter: $x$ and $y$ are taken into consideration, therefore two conditions were created, evaluating the occurrence of a front collision. The first condition requires a reference of $f_{x}$ distance between $m$-segment of $i$-finger relative to the $n$-segment of $j$-finger, to the width of the front section of the two segments (4).

$$
p_{x}=\left|f_{x}\left({ }^{n . i} A_{n . j}\right)\right|-\left(s_{m . i}+s_{n . j}\right)
$$

The second condition concerns the additional length of the front section of the segment, which measured distance $f_{y}$ is relative to.

$$
p_{y}=\left|f_{y}\left({ }^{n . i} A_{n . j}\right)\right|-\left(s_{m . i}+\frac{1_{n . j}+s_{n . j}}{2}\right)
$$

For both equations establishing necessary conditions for the existence of front collision, the orientation of $z$-axis of $m$.i system relative to segment $n . j$ is not important. Occurrence of a collision can be confirmed when the parameter $p_{x}$ or $p_{y}$ assumes a value less than 0 .

\subsection{Side collision}

The last condition for determining the occurrence of a collision between the considered segments is the positive value of the parameter pz according to the Eq. (6). In this case only the widths of the two segments are taken into consideration.

$$
\mathrm{p}_{\mathrm{z}}=\left|\mathrm{f}_{\mathrm{z}}\left({ }^{\mathrm{n} . \mathrm{i}} \mathrm{A}_{\mathrm{n} . \mathrm{j}}\right)\right|-\left(\mathrm{w}_{\mathrm{m} . \mathrm{i}}+\mathrm{w}_{\mathrm{n} . \mathrm{j}}\right)
$$

During the motion control and planning of the segments, it was necessary to determine the motion priority in the selected pair of finger modules. The motion priority condition became critical for the adjacent finger modules. In a situation where a front collision has not yet occurred, and a side collision has been registered, it is possible to continue operation of both modules. Therefore it is obvious, to deny the priority of motion to the finger module, relative to which the possibility of side collision occurrence has been registered.

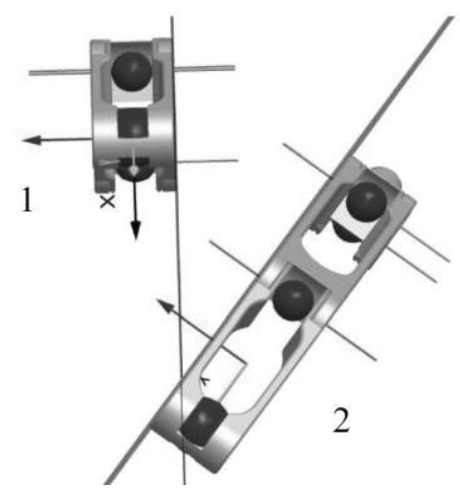

Fig. 5. Unilateral side collision between finger modules

The discussed situation can be seen in figure 5 , in which the finger module 1 does not cause a collision with the finger module 2 , according to the value of the parameter $f_{z}$, while the $f_{z}$ value recalculated for the finger 2 relative to finger 1 this time implicates side collision.
Finally it is possible to determine the last condition allowing the movement in one direction through the use of Heaviside function in equasion (7), where parameter $p_{a}$ is a resultant of $p_{z}$ and a product of $p_{x}$ and $p_{y}$ parameters.

$$
\mathrm{a} \in(\mathrm{x}, \mathrm{y}, \mathrm{z}) \Rightarrow \mathrm{v}_{\mathrm{a}}=\mathrm{H}\left(\mathrm{p}_{\mathrm{a}}\right) \frac{\partial \mathrm{p}_{\mathrm{a}}}{\partial \mathrm{t}}
$$

These collision detection methods allow on a motion planning stage, to assess the possibility of a collision in the following calculation step and in time decide to withdraw the selected finger module if neccessary.

\section{ELIMINATION OF IMPOSSIBLE COLLISIONS}

The process of verification of possible collisions between two selected $4 R$ serial manipulators of established structure, in this case finger modules in Hand-K3 anthropomorhic manipulator, depends on the number of possible collisions. To minimize the time necessary to inspect the possible collisions in any combination, it was crucial to determine the method of exclusion of impossible collision scenerios within searchable combinations. The maximum number of steps needed to verify collision between the three segments of the $i$-finger and 3 modules of $j$-finger is 9 , and because the dependency is bilateral, 18 calculations were required for one pair of figer modules. Therefore, an initial verification was performed at the design stage. Similarly to the procedure while using virtual reality (Zaeh et al., 2004). The first condition for the absence of the collision possibility, concerns parallel orientation of the main planes, in which all 3 segments of each finger module operate. For adjacent finger modules, the comparison of the main planes orientation angle values is sufficient, while the considered pair of modules contains the opposite finger module, it is possible to evaluate their parallelism through comparison of the three values of the $f_{z}$ parameter (Eq. 8). Points $\mathrm{O}, \mathrm{K}$, and $\mathrm{L}$ must be located on a common plane and be included to the segment orientating the main working plane of finger module, relative to which these values are determined.

$$
\mathrm{f}_{\mathrm{z}}\left({ }^{0 . \mathrm{i}} \mathrm{A}_{\mathrm{0} . \mathrm{j}}\right)=\mathrm{f}_{\mathrm{z}}\left({ }^{\mathrm{K} . \mathrm{i}} \mathrm{A}_{\mathrm{0} . \mathrm{j}}\right)=\mathrm{f}_{\mathrm{z}}\left({ }^{\mathrm{L} . \mathrm{i}} \mathrm{A}_{\mathrm{0} . \mathrm{j}}\right)
$$

However, the existence of a collision is still possible for finger modules that have parallel main working planes, when the distance between those planes meet the condition in Eq. 9 .

$\left|\mathrm{f}_{\mathrm{z}}\left({ }^{0 . \mathrm{i}} \mathrm{A}_{\mathrm{0} \mathrm{j}}\right)\right|<\frac{\mathrm{s}_{1 . \mathrm{i}}+\mathrm{s}_{1 . \mathrm{j}}}{2}$

Another condition that eliminates the possibility of a collision, was created in graphical form. The goal of this method is to artbitraly exclude some pairs of mobile segments from the assemblage of possible collisions. Due to the excessive complexity of many variable derivatives calculations required to find the extreme distance between given combination of the segments, the analytical approach was excluded. For this purpose, the knowledge of the workspace, determined by the motion range of segments was used. In the process of collision diagnosis, it is crucial to consider the motion range of joints orienting the main planes of verified modules. Created for this purpose the areas (Fig. 6) represent set of trajectory points of the rotation axis of each segment in the entire motion range of connections orienting the main 
planes of finger modules. These are the longest trajectories available for each module relative to the angle limits of their rotation.

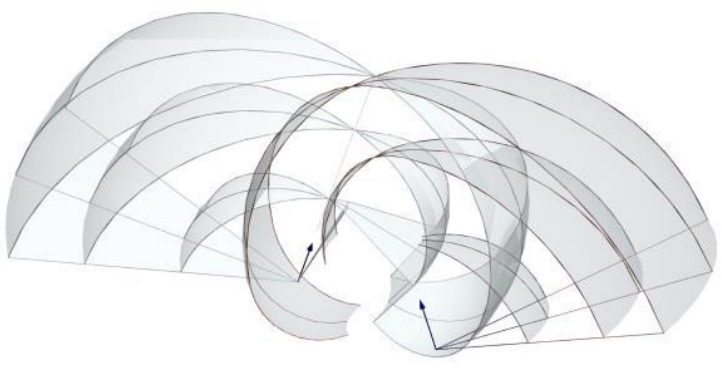

Fig. 6. Workspaces of selected modules of opposing fingers in anthropomorphic manipulator

Because the graphical representation of the workspace in spatial system is not sufficiently clear, an established plane was introduced, on which sets of the trajectories were projected (Fig. 7). This allowed for a simple verification of the possibility of a given pair of segments of collision between each other. In situation where an $t_{n . j}$ trajectory concerning $n$-segment of $j$-finger, intersects with the $a_{m . i}$ workspace of $m$-segment in $i$-finger, a pair of these segments remains in the assemblage of possible collision combinations.

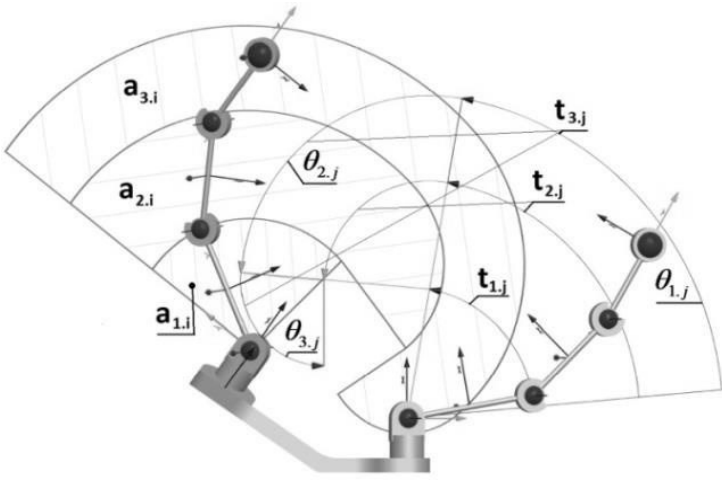

Fig. 7. View of range of finger modules for selected orientation angles of the main working planes

Using the introduced projection plane of the workspaces $a_{1} \div a_{3}$ and the maximal trajectories $t_{1} \div t_{2}$ of all segments in given pair of finger modules, a graphical representation of possible collision assemblage have been acquired (Fig. 8).

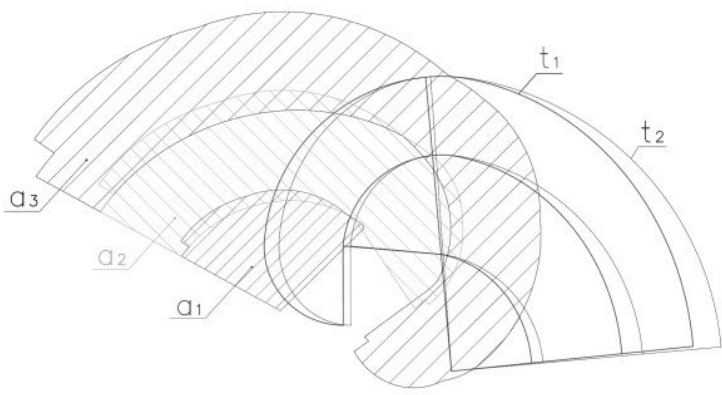

Fig. 8. The total working area $\left(a_{1} \div a_{3}\right)$ of $i$-finger module segments relative to maximal trajectory $\left(t_{1} \div t_{2}\right)$ of $j$-finger module segments
Based on this established graphical representation, the number of calculations performed during the search for potential collisions between segments of the manipulator can be limited through the exclusion of impossible combinations at the design stage. Thus it is possible to minimize the calculation time, needed for the control modules to estimate the trajectory before the further manipulation stages.

\section{CONCLUSIONS}

Presented in this paper methods for assessing the occurrence of collisions between moving segments of anthropomorphic manipulator's finger modules, allowed to increase the effectiveness of movement planning of individual revolute joints by the motion control system. Introducing separate and independent drive control and movement planning computational modules in each finger module based on these methods, allowed to accelerate the operation of the finger modules in the anthropomorphic manipulator, in particular reducing the number of calculations performed at every step of the movement planning. The simplification of established conditions for the collision assessment between moving segments ensured no further increase of drive control units load in the anthropomorphic manipulator.

\section{REFERENCES}

1. Bosscher P., Hedman D.(2011), Real-time collision avoidance algorithm for robotic manipulators, Industrial Robot: An International Journal, Vol. 38, No. 2, 186-197

2. De Luca A., Albu-Schaffer A., Haddadin S., Hirzinger G.(2006),Collision Detection and Safe Reaction with the DLR-III Lightweight Manipulator Arm, Proceedings of the 2006 IEEE/RSJ International Conference on Intelligent Robots and Systems,Beijing

3. Handke A., Twaróg W. (2010), Correlation of sensory parameters' mathematical models with the kinematic structure of a HAND-K3 human hand type manipulator, Acta Mechanica et Automatica,Vol. 4 No. 3.

4. Handke A., Twaróg W. (2012), Method for Plannig the Finger's Movement in the Anthropomorphic Manipulator Hand-K3m Using a Tactile Sensors Network, with the Aim of Optimal Grasping, Mechanism and Machine Science, Vol. 8, 309 - 315.

5. Spencer A., Pryor M., Kapoor C., Tesar D.(2008), Collision Avoidance Techniques for Tele-Operated and Autonomous Manipulators in Overlapping Workspaces, IEEE International Conference on Robotics and Automation, Pasadena.

6. Zaeh M.F., Egermeier H., Petzold B., Spitzweg M. (2004), Dexterous Object Manipulation in a Physics Based Virtual Environment, Proceedings of Mechatronics \& Robotics 2004, Aachen, 1340-1344.

Acknowledgments: The research work reported here was developed within the research project: Prehensile parameters and efficiency studies of the Hand-K3 manipulator prototype (N N514 676740) founded by the National Science Center in Krakow. 